\title{
Ree geometries
}

\section{Fabienne Haot, Koen Struyve and Hendrik Van Maldeghem}

\author{
Communicated by Karl Strambach
}

\begin{abstract}
We introduce a rank 3 geometry for any Ree group over a not necessarily perfect field and show that its full collineation group is the automorphism group of the corresponding Ree group. A similar result holds for two rank 2 geometries obtained as a truncation of this rank 3 geometry. As an application, we show that a polarity in any Moufang generalized hexagon is unambiguously determined by its set of absolute points, or equivalently, its set of absolute lines.
\end{abstract}

Keywords. Ree groups, Moufang sets, Ree unital, mixed hexagons.

2000 Mathematics Subject Classification. 20B25, 51E05, 51E12.

\section{Introduction}

The Ree groups in characteristic 3 (defined by Ree in [6]) and their generalizations over non-perfect fields (by Tits [11]) provide examples of rank 1 groups, or Moufang sets, or split BN-pairs of rank 1 that behave roughly as simple algebraic groups of rank 1, but over a one-dimensional root system consisting of six roots, since the unipotent radicals have nilpotency class 3 . This is a rather rare phenomenon; indeed, until recently, these were the only known rank 1 groups with this property (a second class was discovered and constructed in [5]). Associated with each Ree group is a geometry (called a unital in the finite case), which is a linear space (in the finite case a $2-\left(q^{3}+1, q+1,1\right)$-design), see [4]. This geometry can be viewed as the geometry of involutions in a Ree group, since the blocks are in one-to-one correspondence with a conjugacy class of involutions (in the finite case there is only one conjugacy class). This way, Ree groups can be better understood in that several properties become more geometric and intuitive through this geometry.

In the present paper we introduce another geometry for each Ree group, inspired by the general construction of geometries associated to "wide" rank 1 groups as

The second author is supported by the Fund for Scientific Research - Flanders (FWO - Vlaanderen). The third author is partly supported by a Research Grant of the Fund for Scientific Research Flanders (FWO - Vlaanderen). 
proposed by Tits in one of his lectures; see [15] for an account on this idea ("wide" here means that the unipotent subgroups are not abelian). In fact, this construction is the counterpart for Ree groups of the inversive planes for Suzuki groups (see also [15]). The structure of the geometries that we will introduce is probably slightly more involved than that of the "unitals", but they have the major advantage that the automorphism groups of the corresponding Ree groups are their full automorphism groups (and this is our Main Result below), a result that is not yet proved for the unitals. This result contributes to Tits' programme of characterizing all "wide" rank one groups in this way (i.e., proving a Fundamental Theorem for these geometries). As an application, we can show that every collineation of a Moufang hexagon of mixed type permuting the absolute points of a polarity, centralizes that polarity (or, equivalently, also permutes the absolute lines). This, in turn, means that the set of absolute points of any polarity of any Moufang hexagon (necessarily of mixed type) determines the polarity completely and unambiguously.

The "new" geometries also have a number of interesting combinatorial properties, but we will not concentrate on these, though it would be worthwhile to perform an investigation in that direction.

Every Ree group is the centralizer of a certain outer involution of a Dickson group of type $G_{2}$ over a field of characteristic 3 admitting a Tits endomorphism. A geometric way to see this is to consider the associated Moufang generalized hexagon, which is of mixed type. Then the outer involution is a polarity, and the associated Ree group acts doubly transitively on the absolute points of that polarity. That is exactly the way we are going to define and use the Ree groups. These Moufang hexagons are called Ree hexagons in [14] precisely for that reason.

Hence, in order to investigate the Moufang sets (or rank 1 groups) associated with the Ree groups, we turn to the Ree hexagons, which, as follows from our remarks above, are defined over a field of characteristic 3 admitting a Tits endomorphism $\theta$, and they allow a polarity $\rho$. The absolute points under this polarity, together with the automorphisms of the mixed hexagon commuting with $\rho$, form the Ree-Tits Moufang set. Since we will need an explicit description of the absolute points of $\rho$, we will use coordinates. These will be introduced in Section 2. We define the Ree geometries in Section 4 and state our main results and main application in Section 5 (but we formulate our main results also below in rough terms). The rest of the paper is then devoted to the proofs.

Since the Ree groups have unipotent subgroups of nilpotency class 3 (at least, if the base field is large enough), the Ree geometries that we will define have rank 3 . This means that we will have two types of blocks in our geometry. In this paper we prove that every automorphism of such a geometry is an automorphism of the 
corresponding Ree group, by writing down explicitly the automorphisms of this geometry. But we also do slightly better and prove that the same conclusion holds when restricting to one type of blocks. We call these geometries truncated Ree geometries. Hence, loosely speaking, we may write our main result as follows:

The full automorphism group of a (truncated) Ree geometry is induced by the full collineation group of the corresponding Ree hexagon.

As mentioned above, this is the Fundamental Theorem for the Ree geometries. Other classes of "wide" rank one groups of algebraic origin for which the Fundamental Theorem has been proved are those of type ${ }^{2} A_{2}$ (see [13, 7]), $E_{8,1}$ (unpublished by Tits), $F_{4,1}$ (see [2]), ${ }^{2} B_{2}$ (see [15]), and recently also ${ }^{2} F_{4,\{1,4\}}$ (see [8]; the latter groups are the centralizers of a polarity in an exceptional Moufang quadrangle of type $F_{4}$ ). With the present paper, we $\operatorname{add}^{2} G_{2}$ to that distinguished list.

\section{The coordinatization of the Ree hexagon}

In this section, we present two coordinatizations of the Ree hexagons, which can at the same time serve as a definition of these structures. We start with the coordinatization with respect to one flag $\{(\infty),[\infty]\}$ (a flag is a pair of elements consisting of a point and a line that are incident). This coordinatization was first carried out by De Smet and Van Maldeghem for (finite) generalized hexagons in [3]. For a detailed description of the coordinatization theory for other generalized polygons we refer to [14]. The second coordinatization follows in fact from the natural embedding of the Ree hexagon in $P G(6, \mathbb{K})$.

\subsection{Hexagonal sexternary rings for Ree hexagons}

In [14] a coordinatization theory with respect to a flag $\{(\infty),[\infty]\}$ is described. It is a generalization of Hall's coordinatization for projective planes (which are generalized triangles). Here we describe explicitly the coordinatization of the Ree hexagon. Let $\mathbb{K}$ be a field of characteristic 3 and let $\theta$ be a Tits endomorphism, i.e., $\theta$ is an endomorphism of $\mathbb{K}$ with the property that $\left(x^{\theta}\right)^{\theta}=x^{3}$. Let $\mathbb{K}^{\prime}$ be the image of $\mathbb{K}$ under $\theta$ (note that $\mathbb{K}^{3} \leq \mathbb{K}^{\prime} \leq \mathbb{K}$ ). We define a hexagonal sexternary $\operatorname{ring} \mathcal{R}=\left(\mathbb{K}, \mathbb{K}^{\prime}, \Psi_{1}, \Psi_{2}, \Psi_{3}, \Psi_{4}\right)$ with

$$
\left\{\begin{array}{l}
\Psi_{1}\left(k, a, l, a^{\prime}, l^{\prime}, a^{\prime \prime}\right)=a^{3} k+l, \\
\Psi_{2}\left(k, a, l, a^{\prime}, l^{\prime}, a^{\prime \prime}\right)=a^{2} k+a^{\prime}+a a^{\prime \prime}, \\
\Psi_{3}\left(k, a, l, a^{\prime}, l^{\prime}, a^{\prime \prime}\right)=a^{3} k^{2}+l^{\prime}+k l, \\
\Psi_{4}\left(k, a, l, a^{\prime}, l^{\prime}, a^{\prime \prime}\right)=-a k+a^{\prime \prime},
\end{array}\right.
$$


where $a, a^{\prime}, a^{\prime \prime} \in \mathbb{K}$ and $k, l, l^{\prime} \in \mathbb{K}^{\prime}$. This defines the Ree hexagon $\mathrm{H}\left(\mathbb{K}, \mathbb{K}^{\prime}\right)$ as follows. The points and lines are the $i$-tuples of elements of $\mathbb{K} \cup \mathbb{K}^{\prime}(i \leq 5)$ with alternately an entry in $\mathbb{K}$ and one in $\mathbb{K}^{\prime}$, and for points (lines) the last entry is supposed to be in $\mathbb{K}\left(\mathbb{K}^{\prime}\right)$, except when $i=0$, in case we denote the point by $(\infty)$ and the line by $[\infty]$ (we generally use round parentheses for points and square brackets for lines). Incidence is defined as follows:

- If the number of coordinates of a point $p$ differs by at least 2 from the number of coordinates of a line $L$, then $p$ and $L$ are not incident.

- If the number $i_{p}$ of coordinates of a point $p$ differs by exactly 1 from the number $i_{L}$ of coordinates of a line $L$, then $p$ is incident with $L$ if and only if $p$ and $L$ share the first $i$ coordinates, where $i$ is the smallest among $i_{p}$ and $i_{L}$.

- If $i_{p}=i_{L} \neq 5$, then $p$ is incident with $L$ if and only if $p=(\infty)$ and $L=[\infty]$.

- A point $p$ with coordinates $\left(a, l, a^{\prime}, l^{\prime}, a^{\prime \prime}\right)$ is incident with a line with coordinates $\left[k, b, k^{\prime}, b^{\prime}, k^{\prime \prime}\right]$ (with above notation, and with $b, b^{\prime} \in \mathbb{K}$ and $\left.k^{\prime}, k^{\prime \prime} \in \mathbb{K}^{\prime}\right)$ if and only if

$$
\left\{\begin{array}{l}
\Psi_{1}\left(k, a, l, a^{\prime}, l^{\prime}, a^{\prime \prime}\right)=k^{\prime \prime}, \\
\Psi_{2}\left(k, a, l, a^{\prime}, l^{\prime}, a^{\prime \prime}\right)=b^{\prime \prime}, \\
\Psi_{3}\left(k, a, l, a^{\prime}, l^{\prime}, a^{\prime \prime}\right)=k^{\prime}, \\
\Psi_{4}\left(k, a, l, a^{\prime}, l^{\prime}, a^{\prime \prime}\right)=b^{\prime} .
\end{array}\right.
$$

In general, a generalized hexagon is a point-line structure with the property that each pair of elements (en element can be a point, a line or a flag - the latter is an incident point-line pair) is contained in an ordinary hexagon, but never in an ordinary pentagon, quadrangle, triangle or digon. For a general introduction to generalized hexagons, we refer to [14]. We mention that generalized hexagons and more general, generalized polygons - were introduced by Jacques Tits [10].

Roughly, a Ree group is the centralizer of a polarity in a Ree hexagon, and it acts on a Ree-Tits ovoid (namely, the set of absolute points of the polarity). A polarity is an involutive incidence preserving bijection between points and lines, and between lines and points. A point or line incident with its image is called absolute. An ovoid of a generalized hexagon is a set of mutually opposite points (i.e., points at distance 6 in the incidence graph) such that each point not in the ovoid is collinear to exactly one point of the ovoid. The dual notion is a spread. It is a general fact that the set of absolute points (lines) of a polarity is an ovoid (spread), see Chapter 7 of [14]. 


\subsection{The embedding of the Ree hexagon in $P G(6, \mathbb{K})$}

The Ree hexagon has a natural full embedding in $P G(6, \mathbb{K})$ (here, full means that all points of $P G(6, \mathbb{K})$ incident with a line of the Ree hexagon are points of the Ree hexagon). Indeed, $H\left(\mathbb{K}, \mathbb{K}^{\prime}\right)$ is a substructure of the split Cayley hexagon $H(\mathbb{K})$, which has itself a natural embedding in $P G(6, \mathbb{K})$ as discovered and described by Tits in [10], see also Chapter 2 of [14]. Here, we content ourselves with the table translating the above coordinates to the projective coordinates. We refer to Chapter 3 of [14] for details and proofs.

We write $\alpha$ for $-a l^{\prime}+a^{\prime 2}+a^{\prime \prime} l+a a^{\prime} a^{\prime \prime}$ and $\beta$ for $l-a a^{\prime}-a^{2} a^{\prime \prime}$.

\begin{tabular}{|l|r|}
\hline Coordinates in $\mathrm{H}\left(\mathbb{K}, \mathbb{K}^{\prime}\right)$ & Coordinates in $\mathrm{PG}(6, \mathbb{K})$ \\
\hline$(\infty)$ & $(1,0,0,0,0,0,0)$ \\
$(a)$ & $(a, 0,0,0,0,0,1)$ \\
$(k, b)$ & $(b, 0,0,0,0,1,-k)$ \\
$\left(a, l, a^{\prime}\right)$ & $\left(-l-a a^{\prime}, 1,0,-a, 0, a^{2},-a^{\prime}\right)$ \\
$\left(k, b, k^{\prime}, b^{\prime}\right)$ & $\left(k^{\prime}+b b^{\prime}, k, 1, b, 0, b^{\prime}, b^{2}-b^{\prime} k\right)$ \\
$\left(a, l, a^{\prime}, l^{\prime}, a^{\prime \prime}\right)$ & $\left(\alpha,-a^{\prime \prime},-a,-a^{\prime}+a a^{\prime \prime}, 1, \beta,-l^{\prime}+a^{\prime} a^{\prime \prime}\right)$ \\
\hline
\end{tabular}

\begin{tabular}{|l|r|}
\hline Coordinates in $\mathrm{H}\left(\mathbb{K}, \mathbb{K}^{\prime}\right)$ & Points generating this line \\
\hline$[\infty]$ & $(\infty)$ and $(0)$ \\
{$[k]$} & $(\infty)$ and $(k, 0)$ \\
{$[a, l]$} & $(a)$ and $(a, l, 0)$ \\
{$\left[k, b, k^{\prime}\right]$} & $(k, b)$ and $(k, b, 0)$ \\
{$\left[a, l, a^{\prime}, l^{\prime}\right]$} & $\left(a, l, a^{\prime}\right)$ and $\left(a, l, a^{\prime}, l^{\prime}, 0\right)$ \\
{$\left[k, b, k^{\prime}, b^{\prime}, k^{\prime \prime}\right]$} & $\left(k, b, k^{\prime}, b^{\prime}\right)$ and $\left(0, k^{\prime \prime}, b^{\prime}, k^{\prime}+k k^{\prime \prime}, b\right)$ \\
\hline
\end{tabular}

The subgroup of $\mathrm{PSL}_{7}(\mathbb{K})$ stabilizing the point set and line set of $H\left(\mathbb{K}, \mathbb{K}^{\prime}\right)$ is denoted by $G_{2}\left(\mathbb{K}, \mathbb{K}^{\prime}\right.$ ) and is simple (a mixed group of type $G_{2}$, see [12]).

\section{The Ree-Tits ovoid}

We start from the Ree hexagon $\mathrm{H}\left(\mathbb{K}, \mathbb{K}^{\prime}\right)$, where $\mathbb{K}^{\prime}=\mathbb{K}^{\theta}$, with $\theta$ as above a Tits-endomorphism of $\mathbb{K}$. There is a natural bijective correspondence between the conjugacy classes of Tits-endomorphisms of $\mathbb{K}$ mapping $\mathbb{K}$ onto $\mathbb{K}^{\prime}$ and conjugacy classes of polarities of $H\left(\mathbb{K}, \mathbb{K}^{\prime}\right)$, see Chapter 7 of [14]. It is possible to choose a polarity $\rho$ naturally associated with $\theta$ under the above correspondence such that it fixes the flags $\{(\infty)$, $[\infty]\}$ and $\{(0,0,0,0,0),[0,0,0,0,0]\}$, maps the point (1) 
onto the line [1], and such that it has the following actions on points and lines with five coordinates:

$$
\begin{aligned}
\left(a, l, a^{\prime}, l^{\prime}, a^{\prime \prime}\right)^{\rho} & =\left[a^{\theta}, l^{\theta^{-1}}, a^{\prime \theta}, l^{\prime \theta^{-1}}, a^{\prime \prime \theta}\right], \\
{\left[k, b, k^{\prime}, b^{\prime}, k^{\prime \prime}\right]^{\rho} } & =\left(k^{\theta^{-1}}, b^{\theta}, k^{\theta^{-1}}, b^{\prime \theta}, k^{\prime \prime \theta^{-1}}\right),
\end{aligned}
$$

for all $a, a^{\prime}, a^{\prime \prime}, b, b^{\prime} \in \mathbb{K}$ and $k, k^{\prime}, k^{\prime \prime}, l, l^{\prime} \in \mathbb{K}^{\prime}$. Moreover, this action determines the images of all other points and lines, see again Chapter 7 of [14]. The absolute points under this polarity form an ovoid of the Ree hexagon: the Ree-Tits ovoid, see Chapter 7 of [14]. Now the point $\left(a, l, a^{\prime}, l^{\prime}, a^{\prime \prime}\right)$ is absolute for $\rho$ if and only if it is incident with its image. This leads to the following conditions:

$$
\left\{\begin{array}{l}
l=a^{\prime \prime \theta}-a^{\theta+3}, \\
l^{\prime}=a^{2 \theta+3}+a^{\prime \theta}+a^{\theta} a^{\prime \prime \theta} .
\end{array}\right.
$$

Coordinates of the Ree-Tits ovoid in PG(6, $\mathbb{K})$. We write $\left(a, a^{\prime \prime}, a^{\prime}-a a^{\prime \prime}\right)$ as a shorthand notation for the absolute point with coordinates $\left(a, a^{\prime \prime \theta}-a^{3+\theta}, a^{\prime}\right.$, $\left.a^{3+2 \theta}+a^{\prime \theta}+a^{\theta} a^{\prime \prime \theta}, a^{\prime \prime}\right)$. Hence the mapping

$$
\mathbb{K}^{3} \rightarrow \mathbb{K}^{5}:\left(a, a^{\prime}, a^{\prime \prime}\right) \mapsto\left(a, a^{\prime \theta}-a^{3+\theta}, a a^{\prime}+a^{\prime \prime}, a^{3+2 \theta}+a^{\prime \prime \theta}-a^{\theta} a^{\prime \theta}, a^{\prime}\right)
$$

identifies $\mathbb{K}^{3}$ with the set of absolute points distinct from $(\infty)$. Now, for $a, a^{\prime}, a^{\prime \prime} \in$ $\mathbb{K}$, we put

$$
\begin{aligned}
& f_{1}\left(a, a^{\prime}, a^{\prime \prime}\right)=-a^{4+2 \theta}-a a^{\prime \prime \theta}+a^{1+\theta} a^{\prime \theta}+a^{\prime \prime 2}+a^{1+\theta}-a^{\prime} a^{3+\theta}-a^{2} a^{\prime 2}, \\
& f_{2}\left(a, a^{\prime}, a^{\prime \prime}\right)=-a^{3+\theta}+a^{\prime \theta}-a a^{\prime \prime}+a^{2} a^{\prime}, \\
& f_{3}\left(a, a^{\prime}, a^{\prime \prime}\right)=-a^{3+2 \theta}-a^{\prime \prime \theta}+a^{\theta} a^{\prime \theta}+a^{\prime} a^{\prime \prime}+a a^{\prime 2} .
\end{aligned}
$$

So the set of absolute points can be described in $P G(6, \mathbb{K})$ by

$$
\begin{aligned}
\Omega=\{(1,0,0,0,0,0,0)\} & \cup\left\{\left(f_{1}\left(a, a^{\prime}, a^{\prime \prime}\right),-a^{\prime},-a,-a^{\prime \prime}, 1,\right.\right. \\
& \left.\left.f_{2}\left(a, a^{\prime}, a^{\prime \prime}\right), f_{3}\left(a, a^{\prime}, a^{\prime \prime}\right)\right) \mid a, a^{\prime}, a^{\prime \prime} \in \mathbb{K}\right\} .
\end{aligned}
$$

Compact notation. As before, we associate the triple $\left(a, a^{\prime \prime}, a^{\prime}-a a^{\prime \prime}\right)$ with the point $\left(a, a^{\prime \prime \theta}-a^{3+\theta}, a^{\prime}, a^{3+2 \theta}+a^{\prime \theta}+a^{\theta} a^{\prime \prime \theta}\right)$. The set of absolute points under the polarity is now

$$
\Omega=\{(\infty)\} \cup\left\{\left(a, a^{\prime}, a^{\prime \prime}\right) \mid a, a^{\prime}, a^{\prime \prime} \in \mathbb{K}\right\} .
$$

The elements of the root group $U_{\infty}$ (a root group is a unipotent subgroup) of the ovoid fixing the point $(\infty)$ act as follows on the remaining points $\left(x, x^{\prime}, x^{\prime \prime}\right)$ : the unipotent element that fixes $(\infty)$ and maps $(0,0,0)$ to $\left(y, y^{\prime}, y^{\prime \prime}\right)$ maps $\left(x, x^{\prime}, x^{\prime \prime}\right)$ 
to

$$
\left(x, x^{\prime}, x^{\prime \prime}\right) \cdot\left(y, y^{\prime}, y^{\prime \prime}\right)=\left(x+y, x^{\prime}+y^{\prime}+x y^{\theta}, x^{\prime \prime}+y^{\prime \prime}+x y^{\prime}-x^{\prime} y-x y^{\theta+1}\right),
$$

and this action can also be seen as the multiplication inside $U_{\infty}$, see Chapter 7 of [14].

We obtain the Ree-Tits Moufang set. Formally, a Moufang set is a set $X$ together with a family of (root) groups $\left(U_{x}\right)_{x \in X}$ such that $U_{x}$ acts sharply transitively on $X \backslash\{x\}$ and fixes $x$, and such that each $U_{y}, y \in X$, acts by conjugation on the set $\left\{U_{x} \mid x \in X\right\}$. The (simple) Ree groups arise as (simple subgroups of the) centralizers of polarities in these hexagons. More exactly, the Ree group $\mathrm{R}(\mathbb{K}, \theta)$ is defined as the centralizer in $\mathrm{G}_{2}\left(\mathbb{K}, \mathbb{K}^{\prime}\right)$ of the outer automorphism $\rho$. This group is simple if $|\mathbb{K}|>3$ and the multiplicative group of $\mathbb{K}$ is generated by all squares together with -1 , see [6]. In any case, the group generated by the root groups is simple, provided $|\mathbb{K}|>3$, and it coincides with the derived group $\mathbf{R}^{\prime}(\mathbb{K}, \theta)$. For $|\mathbb{K}|=3, R(\mathbb{K}, \theta)=R(3)$ is isomorphic to $P \Gamma L_{2}(8)$ and contains $\mathrm{PSL}_{2}(8)$ as a simple subgroup of index 3 .

We can see the Ree-Tits ovoid and its automorphism group embedded in the Ree hexagon as a representation of the Ree-Tits Moufang set. Henceforth, we will denote by $\Omega$ the Ree-Tits ovoid, and by $U_{x}, x \in \Omega$, the root group fixing $x$ in the Ree-Tits Moufang set over the field $\mathbb{K}$ with associated Tits endomorphism $\theta$.

We will also need the explicit form of a generic element of the root group $U_{(0,0,0)}$, which we shall briefly denote by $U_{0}$. This is best given by the action on coordinates in the projective space. Such generic element $u_{\left(x, x^{\prime}, x^{\prime \prime}\right)}^{(0,0,0)}$ then looks like (and $x, x^{\prime}, x^{\prime \prime}$ are arbitrary in $\mathbb{K}$ ):

$$
\begin{aligned}
\bar{x}=\left(\begin{array}{llllll}
x_{0} x_{1} & x_{2} & x_{3} & x_{4} & x_{5} & x_{6}
\end{array}\right) \\
\mapsto \bar{x} \cdot\left(\begin{array}{ccccccc}
1 & f_{2}\left(x, x^{\prime}, x^{\prime \prime}\right) & f_{3}\left(x, x^{\prime}, x^{\prime \prime}\right) & x^{\prime \prime} & f_{1}\left(x, x^{\prime}, x^{\prime \prime}\right)-x^{\prime}-x \\
0 & 1 & -x^{\theta} & 0 & x^{\prime}-x^{1+\theta} & 0 & 0 \\
0 & 0 & 1 & 0 & x & 0 & 0 \\
0 & -x & x^{\prime} & 1 & -x^{\prime \prime} & 0 & 0 \\
0 & 0 & -x^{\prime \prime}-x x^{\prime} & x & 1 & 0 & 0 \\
0 & x^{2} & -x^{\prime}-x^{\prime} & p & 1 & 0 \\
0 & r & s & -x^{\prime}+x^{1+\theta} & q & x^{\theta} & 1
\end{array}\right),
\end{aligned}
$$

where

$$
\left\{\begin{array}{l}
p=x^{3+\theta}-x^{\prime \theta}-x x^{\prime \prime}-x^{2} x^{\prime} \\
q=x^{\prime \prime} \theta+x^{\theta} x^{\prime \theta}-x x^{\prime 2}-x^{2+\theta} x^{\prime}-x^{1+\theta} x^{\prime \prime}-x^{3+2 \theta} \\
r=x^{\prime \prime}-x x^{\prime}+x^{2+\theta} \\
s=x^{\prime 2}-x^{1+\theta} x^{\prime}-x^{\theta} x^{\prime \prime}
\end{array}\right.
$$

see Section 9.2.4 of [9].

We are now ready to define the Ree geometries. 


\section{The Ree geometry}

As already mentioned, the Ree groups have root groups of nilpotency class 3. As a consequence, the geometries that we will define corresponding to the Ree groups will have rank 3 . This means that we will have two types of blocks in our geometry and that blocks of one type are subsets of the others. In order to distinguish the two types of blocks, we will call the "smallest" ones circles, and the others spheres.

The point set $\mathcal{P}$ of our Ree geometry $\mathscr{E}=(\mathcal{P}, \mathcal{B})$ is the Ree-Tits ovoid. The circles arise as orbits of a point $y$ under the center $Z\left(U_{x}\right)$ for some point $x \in$ $\mathcal{P} \backslash\{y\}$, together with that point $x$. This particular point $x$ is then called a gnarl of this circle. So every point and gnarl defines a circle in a unique way. The spheres are again a point $x$ together with the orbit of some point $y, y \neq x$, but this time under the group $U_{x}^{\prime}=\left[U_{x}, U_{x}\right]$. The point $x$ is a gnarl of the sphere. The circles and spheres together form the block set $\mathscr{B}$ of $\mathscr{E}$. The set of circles will be denoted by $\mathcal{C}$, and the set of spheres by $\mathcal{~}$. We can define two further geometries by restricting the set of blocks. We call the geometries $\mathscr{E}_{\mathcal{C}}=(\mathcal{P}, \mathcal{C})$ and $\mathscr{E}_{\mathscr{\delta}}=(\mathcal{P}, \mathcal{S})$ the truncated Ree geometries. The gnarls of circles and spheres will turn out to be unique.

Let us be more concrete now and look for the coordinates of the circles and spheres which have $(\infty)$ for gnarl.

We first claim that, if $|\mathbb{K}|>3$, then the group $U_{\infty}^{\prime}$ is precisely $\left\{\left(0, u^{\prime}, u^{\prime \prime}\right) \mid\right.$ $\left.u^{\prime}, u^{\prime \prime} \in \mathbb{K}\right\}$. Indeed, computing an arbitrary commutator, we get

$$
\begin{aligned}
{\left[\left(u_{1}, u_{1}^{\prime}, u_{1}^{\prime \prime}\right),\right.} & \left.\left(u_{2}, u_{2}^{\prime}, u_{2}^{\prime \prime}\right)\right] \\
= & \left(0, u_{1} u_{2}^{\theta}-u_{2} u_{1}^{\theta}, u_{1}^{\prime} u_{2}-u_{1} u_{2}^{\prime}-u_{1} u_{2}^{1+\theta}+u_{2} u_{1}^{1+\theta}\right) .
\end{aligned}
$$

Noting that $\left(0, x^{\prime}, 0\right) \cdot\left(0,0, x^{\prime \prime}\right)=\left(0, x^{\prime}, x^{\prime \prime}\right)$, we only have to show that $\left(0, x^{\prime}, 0\right) \in U_{\infty}^{\prime}$, for all $x^{\prime} \in \mathbb{K}$, and that $\left(0,0, x^{\prime \prime}\right) \in U_{\infty}^{\prime}$, for all $x^{\prime \prime} \in \mathbb{K}$. Putting $u_{1}=u_{1}^{\prime \prime}=u_{2}^{\prime}=u_{2}^{\prime \prime}=0, u_{1}^{\prime}=1$ and $u_{2}=x^{\prime \prime}$ in the above commutator, we see that $\left(0,0, x^{\prime \prime}\right) \in U_{\infty}$. Now let $x^{\prime} \in \mathbb{K}$ be arbitrary. Since $|\mathbb{K}|>3$, there exists an element $t \in \mathbb{K}$ with $t^{3}-t \neq 0$. Put $k=t^{3}-t$ and let $y=x^{\prime} k^{-\theta}$. Putting $u_{1}^{\prime}=$ $u_{2}^{\prime}=u_{1}^{\prime \prime}=u_{2}^{\prime \prime}=0$ and $\left(u_{1}, u_{2}\right)=\left(y, t^{3}\right)$, respectively $\left(u_{1}, u_{2}\right)=\left(t^{\theta} y, 1\right)$, we obtain $\left(0, t^{3 \theta} y-t^{3} y^{\theta}, 0\right) \in U_{\infty}^{\prime}$ and $\left(0, t^{\theta} y-t^{3} y^{\theta}, 0\right) \in U_{\infty}^{\prime}$. Multiplying the former with the inverse of the latter, we see that $\left(0, x^{\prime}, 0\right) \in U_{\infty}^{\prime}$, proving our claim.

If $|\mathbb{K}|=3$, then $U_{\infty}^{\prime}$ has order 3 and coincides with the center (see below). In this case, we will substitute $U_{x}^{\prime}$ by the subgroup of $U_{x}$ generated by the elements of order 3 , and we will denote it, with abuse of notation, by $U_{x}^{\prime}$ (but there will be no confusion possible), since for $|\mathbb{K}|>3$, the derived group coincides with the group generated by elements of order 3 (as one can check easily). 
The center of $U_{\infty}$ is the subgroup $\left\{\left(0,0, u^{\prime \prime}\right) \mid u^{\prime \prime} \in \mathbb{K}\right\}$. Indeed, this follows from the explicit form of the multiplication in $U_{\infty}$ by standard arguments. Since the commutator of an element $\left(0, u_{1}^{\prime}, u_{1}^{\prime \prime}\right) \in U_{\infty}^{\prime}$ and $\left(u_{2}, u_{2}^{\prime}, u_{2}^{\prime \prime}\right) \in U_{\infty}$ is

$$
\begin{aligned}
{\left[\left(0, u_{1}^{\prime}, u_{1}^{\prime \prime}\right),\left(u_{2}, u_{2}^{\prime}, u_{2}^{\prime \prime}\right)\right] } & =\left(0,0, u_{1}^{\prime} u_{2}\right) \\
& =\left(0,0, u^{\prime \prime}\right),
\end{aligned}
$$

with $u^{\prime \prime}$ essentially arbitrary, we see that the second derived group $U_{\infty}^{\prime \prime}$ coincides with the center $Z\left(U_{\infty}\right)$.

Now, since the circles having $(\infty)$ as gnarl are the orbits of a point $\left(a, a^{\prime}, a^{\prime \prime}\right)$ under the group $\{(0,0, x) \mid x \in \mathbb{K}\}$, all circles with $(\infty)$ as gnarl are given by

$$
\left\{\left(a, a^{\prime}, a^{\prime \prime}+x\right) \mid x \in \mathbb{K}\right\} \cup\{(\infty)\}=\left\{\left(a, a^{\prime}, t\right) \mid t \in \mathbb{K}\right\} \cup\{(\infty)\} .
$$

The spheres with gnarl $(\infty)$ have the following description:

$$
\begin{gathered}
\left\{a, a^{\prime}+x^{\prime}, a^{\prime \prime}+x^{\prime \prime}+a x^{\prime} \mid x^{\prime}, x^{\prime \prime} \in \mathbb{K}\right\} \cup\{(\infty)\} \\
=\left\{\left(a, t^{\prime}, t^{\prime \prime}\right) \mid t^{\prime}, t^{\prime \prime} \in \mathbb{K}\right\} \cup\{(\infty)\} .
\end{gathered}
$$

We can now interpret the algebraic description of a circle and a sphere with gnarl $(\infty)$ in the corresponding Ree hexagon $H\left(\mathbb{K}, \mathbb{K}^{\prime}\right)$. We leave it as an easy exercise to the reader to see that these circles and spheres look as follows: a circle is the set of absolute points at distance 3 from a non-absolute line $M$, not going through an absolute point. The unique absolute point for which its corresponding absolute line intersects $M$ is the gnarl of the circle. Likewise, a sphere is the set of absolute points not opposite some non-absolute point $p$, with $p$ lying on an absolute line. The unique absolute point at distance 2 from $p$ is the gnarl of the sphere. Conversely, every such set is a circle or sphere, respectively. It follows now easily that the gnarl of a circle and of a sphere is unique. These gnarls will play a prominent role in our proofs.
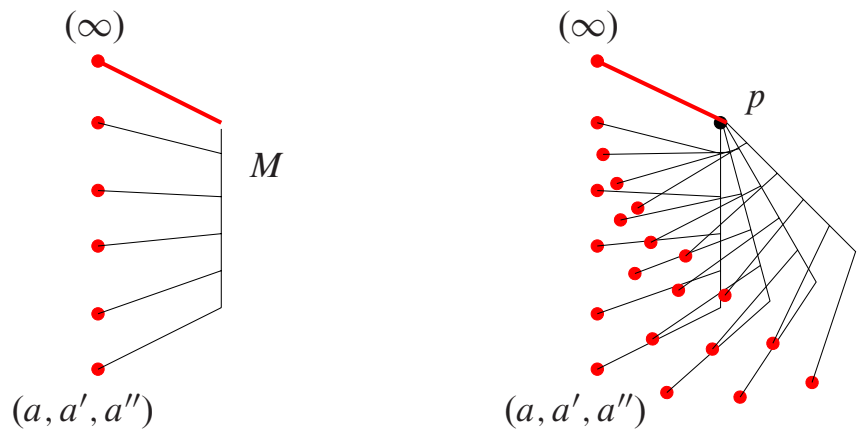
As an application we make the following important observation.

Lemma 4.1. A sphere contains only circles with the same gnarl. Also, the point set of a sphere, except for its gnarl, is partitioned by the circles contained in the sphere.

Proof. Let us consider a sphere and circle, and assume that this sphere's gnarl is the absolute point $p$ while the gnarl of the circle is a different absolute point $q$. The flags $\left\{p, p^{\rho}\right\}$ and $\left\{q, q^{\rho}\right\}$ determine an unique apartment $\Sigma$ containing both flags, and because both flags are absolute $\rho$ will stabilize $\Sigma$. Denote the unique line in $\Sigma$ at distance 2 from $q^{\rho}$ and at distance 3 from $p$ with $L$ and the projection of $q$ on $p^{\rho}$ with $r$. Let $a$ be a third absolute point on the circle different from both $p$ and $q$.

Because $a$ lies on the circle with gnarl $q$ through $p, a$ lies at distance 3 from $L$. Similarly $a$ also lies at distance 4 from $r$ because of the definition of a sphere. The last statement implies that $a^{\rho}$ lies at distance 4 from the line $r^{\rho}$. This line $r^{\rho}$ intersects the line $L$, so the point $a$ and the lines $L, r^{\rho}, a^{\rho}$ are contained in a ordinary 5-gon, which contradicts the definition of a generalized hexagon. This proves the first assertion.

For the second assertion, we just consider the circles defined by the non-absolute lines of $H\left(\mathbb{K}, \mathbb{K}^{\theta}\right)$ through the point defining the sphere in question.

\section{Statement of main results}

Given the construction of the circles and spheres in the corresponding Ree hexagon $H\left(\mathbb{K}, \mathbb{K}^{\theta}\right)$, it is clear that every collineation of $H\left(\mathbb{K}, \mathbb{K}^{\theta}\right)$ that commutes with the polarity $\rho$ induces a collineation of the Ree geometry and its truncations. Our main results now say that also the converse holds. More precisely:

Main Result 1. The full automorphism group of the Ree geometry $\mathcal{E}=(\mathcal{P}, \mathscr{B})$ is the centralizer of $\rho$ in the full collineation group of $\mathrm{H}\left(\mathbb{K}, \mathbb{K}^{\theta}\right)$.

Likewise, we will show:

Main Result 2. The full automorphism groups of the truncated Ree geometries $\mathscr{E}_{e}=(\mathcal{P}, \mathcal{C})$ and $\mathscr{G}_{\mathcal{S}}=(\mathcal{P}, \mathcal{S})$ coincide with the centralizer of $\rho$ in the full collineation group of $\mathrm{H}\left(\mathbb{K}, \mathbb{K}^{\theta}\right)$.

As a main consequence we will be able to show:

Main Corollary. The stabilizer of a Ree-Tits ovoid in the full collineation group of $\mathrm{H}\left(\mathbb{K}, \mathbb{K}^{\theta}\right)$ coincides with the centralizer of the corresponding polarity in the full 
collineation group of $\mathrm{H}\left(\mathbb{K}, \mathbb{K}^{\theta}\right)$. Consequently, any polarity is determined by its set of absolute points.

The latter was already announced in [14] as Theorem 7.7.9, but not proved there.

Another consequence is the following. We denote by $G$ the centralizer of $\rho$ in the full collineation group of $\mathrm{H}\left(\mathbb{K}, \mathbb{K}^{\theta}\right)$, and $G^{\dagger}$ denotes the simple subgroup of $G$ generated by the unipotent elements (or, equivalently, the last term in the derived series of $G$ ).

Second Corollary. The full automorphism group of the Moufang set naturally associated with $G$ is $G$ itself. Also, the full automorphism group of $G^{\dagger}$ is $G$.

The first assertion is direct consequence of Main Result 1 and the second assertion follows immediately from the first by using the uniqueness of the unipotent subgroups of $G$, as proved in [1]. As far as we are aware of, this has not been observed before in full generality, certainly not for the imperfect case (in the finite case everything follows from the classification of finite simple groups, of course).

We will now prove these results.

\section{Auxiliary tools}

\subsection{The derived geometry at $(\infty)$}

We define the structure $\mathcal{E}^{\prime}=\left(\mathcal{P}^{\prime}, \mathcal{B}^{\prime}\right)$, where $\mathcal{P}^{\prime}=\mathcal{P} \backslash\{(\infty)\}$, and $\mathscr{B}^{\prime}$ is the set of blocks of $\mathcal{E}$ going through $(\infty)$, with $(\infty)$ removed. We call this the derived geometry at $(\infty)$, inspired by a similar concept in the theory of designs. In order to know the coordinates of the circles through $(\infty)$ we first write down the coordinates of the circles with gnarl $(\infty)$. As we saw earlier, these are the sets

$$
\left\{\left(a, a^{\prime}, t\right) \mid t \in \mathbb{K}\right\} \cup\{(\infty)\}, \quad \text { with } a, a^{\prime} \in \mathbb{K} .
$$

Removing the point $(\infty)$ gives us the vertical line $L_{a, a^{\prime}}$. We now compute the coordinates of the circle with gnarl $(0,0,0)$ through $(\infty)$. The point $(\infty)$ is identified with $(1,0,0,0,0,0,0)$, so its orbit under $Z\left(U_{0}\right)$ (using the elements $u_{\left(0,0, x^{\prime \prime}\right)}^{(0,0,0)}$ defined above) is the set

$$
\begin{gathered}
\left\{\left(1, f_{2}\left(0,0, x^{\prime \prime}\right), f_{3}\left(0,0, x^{\prime \prime}\right), x^{\prime \prime}, f_{1}\left(0,0, x^{\prime \prime}\right), 0,0\right) \mid x^{\prime \prime} \in \mathbb{K}\right\} \\
=\left\{\left(1,0,-x^{\prime \prime \theta}, x^{\prime \prime}, x^{\prime \prime 2}, 0,0\right) \mid x^{\prime \prime} \in \mathbb{K}\right\} .
\end{gathered}
$$

Putting $x=x^{\prime \prime-2-\theta}$ (and hence $x^{\prime \prime}=x^{-2+\theta}$ ), adding the gnarl and deleting the point $(\infty)$, we obtain the set $\left\{\left(x, 0,-x^{2+\theta}\right) \mid x \in \mathbb{K}\right\}$. The image of this set 
under $\left(a, a^{\prime}, a^{\prime \prime}\right) \in U_{\infty}$ is the set

$$
\left\{\left(a+x, a^{\prime}+a^{\theta} x, a^{\prime \prime}+\left(a^{\prime}-a^{1+\theta}\right) x-x^{2+\theta}\right) \mid x \in \mathbb{K}\right\},
$$

which we call the ordinary line $C_{\left(a, a^{\prime}, a^{\prime \prime}\right)}$ (with $\left.\operatorname{gnarl}\left(a, a^{\prime}, a^{\prime \prime}\right)\right)$. Note that unlike the vertical lines, these are not affine lines.

Just as we did for circles, we consider the spheres with gnarl $(\infty)$ and the other spheres through $(\infty)$ separately.

The spheres with gnarl $(\infty)$ are the sets $\left\{\left(a, t^{\prime}, t^{\prime \prime}\right) \mid t^{\prime}, t^{\prime \prime} \in \mathbb{K}\right\} \cup\{(\infty)\}$, with $a \in \mathbb{K}$. Removing the point $(\infty)$ gives us the vertical plane $P_{a}$.

The orbit of $(\infty)$ under $U_{0}^{\prime}$, using the elements $u_{\left(0, x^{\prime}, x^{\prime \prime}\right)}^{(0,0,0)}$ is the set

$$
\begin{aligned}
& \left\{\left(1, f_{2}\left(0, x^{\prime}, x^{\prime \prime}\right), f_{3}\left(0, x^{\prime}, x^{\prime \prime}\right), x^{\prime \prime}, f_{1}\left(0, x^{\prime}, x^{\prime \prime}\right),-x^{\prime}, 0\right) \mid x^{\prime}, x^{\prime \prime} \in \mathbb{K}\right\} \\
& =\left\{\left(1, x^{\prime \theta},-x^{\prime \prime \theta}+x^{\prime} x^{\prime \prime}, x^{\prime \prime}, x^{\prime \prime 2}+x^{\prime 1+\theta},-x^{\prime}, 0\right) \mid x^{\prime}, x^{\prime \prime} \in \mathbb{K}\right\} \\
& =\left\{\left(\frac{x^{\prime \prime \theta}-x^{\prime} x^{\prime \prime}}{x^{\prime \prime 2}+x^{\prime 1+\theta}}, \frac{-x^{\prime \theta}}{x^{\prime \prime 2}+x^{\prime 1+\theta}}, \frac{-x^{\prime \prime}}{x^{\prime \prime 2}+x^{\prime 1+\theta}}\right) \mid\right. \\
& \left.\mathbb{K} \times \mathbb{K} \ni\left(x^{\prime}, x^{\prime \prime}\right) \neq(0,0)\right\} \cup\{(\infty)\} .
\end{aligned}
$$

Note that $x^{\prime \prime 2} \neq-x^{\prime 1+\theta}$ is equivalent with $\left(x^{\prime}, x^{\prime \prime}\right) \neq(0,0)$. Adding $(0,0,0)$, the image of this sphere under $\left(a, a^{\prime}, a^{\prime \prime}\right) \in U_{\infty}$ is the set

$$
\begin{aligned}
\left\{\left(\frac{x^{\prime \prime \theta}-x^{\prime} x^{\prime \prime}}{x^{\prime \prime 2}+x^{\prime 1+\theta}}, \frac{-x^{\prime \theta}}{x^{\prime \prime 2}+x^{\prime 1+\theta}}, \frac{-x^{\prime \prime}}{x^{\prime \prime 2}+x^{\prime 1+\theta}}\right) \cdot\left(a, a^{\prime}, a^{\prime \prime}\right) \mid\right. \\
\left.\mathbb{K} \times \mathbb{K} \ni\left(x^{\prime}, x^{\prime \prime}\right) \neq(0,0)\right\} \cup\left\{\left(a, a^{\prime}, a^{\prime \prime}\right),(\infty)\right\} .
\end{aligned}
$$

Removing the point $(\infty)$ gives us the ordinary plane $S_{\left(a, a^{\prime}, a^{\prime \prime}\right)}$ (with gnarl $\left(a, a^{\prime}, a^{\prime \prime}\right)$ ). Again note that these are not affine planes, while the vertical planes are.

Notice that points of vertical planes have constant first coordinate, while the points of an ordinary line never have constant first coordinate. This provides an algebraic proof of Lemma 4.1.

\subsection{Parallelism in this derived structure}

We consider the set of points $\left(x, x^{\prime}, x^{\prime \prime}\right)$ as an affine space in the standard way, and call the planes affine planes. We assume that the coordinates are given with respect to a basis with axes $X, Y, Z$.

First we remark that every ordinary line $C_{\left(a, a^{\prime}, a^{\prime \prime}\right)}$ completely lies in the affine plane with equation $Y=a^{\theta} X+\left(a^{\prime}-a^{1+\theta}\right)$. We say that two ordinary lines $C_{1}$ and $C_{2}$ are parallel if all vertical lines intersecting $C_{1}$ intersect $C_{2}$ - in that 
case the two ordinary lines lie in the same affine plane - or if there is no vertical line intersecting both ordinary lines - which implies that the ordinary lines lie in parallel, but disjoint, affine planes.

We claim that two ordinary lines $C_{\left(a, a^{\prime}, a^{\prime \prime}\right)}$ and $C_{\left(b, b^{\prime}, b^{\prime \prime}\right)}$ are parallel if and only if $a=b$. Indeed, a vertical line meeting the ordinary line $C_{\left(a, a^{\prime}, a^{\prime \prime}\right)}$ must lie in the affine plane $Y=a^{\theta} X+\left(a^{\prime}-a^{1+\theta}\right)$, so any vertical line meeting both $C_{\left(a, a^{\prime}, a^{\prime \prime}\right)}$ and $C_{\left(b, b^{\prime}, b^{\prime \prime}\right)}$ must lie in the intersection of

$$
\left\{\begin{array}{l}
Y=a^{\theta} X+\left(a^{\prime}-a^{1+\theta}\right), \\
Y=b^{\theta} X+\left(b^{\prime}-b^{1+\theta}\right) .
\end{array}\right.
$$

This has a unique solution if and only if $a \neq b$, proving our claim.

We have the following direct lemma.

Lemma 6.1. The gnarls of the ordinary lines of the parallel class of $C_{\left(a, a^{\prime}, a^{\prime \prime}\right)}$ are exactly the points of the vertical plane $P_{a}$.

Proof. The above says that the set of gnarls of the lines of the parallel class of $C_{\left(a, a^{\prime}, a^{\prime \prime}\right)}$ is given by $\left\{\left(a, t^{\prime}, t^{\prime \prime}\right) \mid t^{\prime}, t^{\prime \prime} \in \mathbb{K}\right\}$, which is exactly $P_{a}$.

\subsection{Ree unitals}

In Section 10, we will use the Ree unitals mentioned in the introduction. We do not need a formal definition, or a complete description of them, but only the following two facts: (1) two different points are joined by exactly one block of the Ree unital, (2) the block through $(\infty)$ and $\left(a, 0, a^{\prime \prime}\right)$, with $a$ and $a^{\prime \prime} \in \mathbb{K}$, is given by $\{(\infty)\} \cup\left\{\left(a, t, a^{\prime \prime}-a t\right) \mid t \in \mathbb{K}\right\}$ (see Chapter 7 of [14]).

If $B$ is a unital block containing $(\infty)$, then we will call the set $B \backslash\{(\infty)\}$ an affine unital block.

\section{Automorphism group of the Ree geometry}

General idea. We consider an automorphism $\varphi$ of the Ree geometry. Without loss of generality we may assume that $\varphi$ fixes both $(\infty)$ and $(0,0,0)$. We will prove that $\varphi$ must preserve gnarls, and this will imply that it has to preserve the parallelism we just defined. We then compute the algebraic form of $\varphi$ and conclude that it can be extended to $\mathrm{H}\left(\mathbb{K}, \mathbb{K}^{\theta}\right)$.

Lemma 7.1. The automorphism $\varphi$ maps the gnarl of any sphere onto the gnarl of the image of the sphere, and it maps the gnarl of any circle onto the gnarl of the image of the circle under $\varphi$. 
Proof. Any automorphism of $\Delta$ maps spheres onto spheres and circles onto circles, since every circle is properly contained in a sphere, but no sphere is properly contained in any circle or sphere. Since the gnarl of a sphere is exactly the intersection of all circles contained in it (by Lemma 4.1), and there are at least two such circles, $\varphi$ preserves gnarls of spheres. But then $\varphi$ must also preserve the gnarls of these circles.

Since $\varphi$ fixes the points $(\infty)$ and $(0,0,0)$, it acts on the derived structure $\mathcal{E}^{\prime}$, and the previous lemma implies that $\varphi$ fixes the set of vertical lines. Therefore the points $\left(a, a^{\prime}, z_{1}\right)$ and $\left(a, a^{\prime}, z_{2}\right)$ are mapped on the same vertical line. If we represent $\varphi$ as follows:

$$
\varphi:(x, y, z) \mapsto\left(g_{1}(x, y, z), g_{2}(x, y, z), g_{3}(x, y, z)\right),
$$

then both $g_{1}$ and $g_{2}$ have to be independent of $z$ and we write $g_{i}(x, y, z)=$ $g_{i}(x, y), i=1,2$.

The mapping $\varphi$ preserves the parallel relation between ordinary lines, since the number of vertical lines meeting two circles (i.e. none, one or all) is preserved un$\operatorname{der} \varphi$. This translates to $g_{1}$ being independent of $y$. Indeed, two points $\left(a, y_{1}, z_{1}\right)$ and $\left(a, y_{2}, z_{2}\right)$ being the gnarls of two parallel ordinary lines are mapped onto two gnarls of parallel ordinary lines, which implies that $g_{1}\left(a, y_{1}\right)=g_{1}\left(a, y_{2}\right)$ for every choice for $y_{1}$ and $y_{2}$.

The point $(0,0,0)$ is fixed by $\varphi$, so the affine plane $Y=0$ - which is the unique affine plane containing both $C_{(0,0,0)}$ and $L_{0,0}$, and which consist of the union of vertical lines all meeting $C_{(0,0,0)}$ - is fixed by $\varphi$. The plane $Y=c_{1}$ - which is also a union of vertical lines - must necessarily get mapped onto a plane $Y=c_{2}$. So $g_{2}\left(x, c_{1}\right)=g_{2}\left(0, c_{1}\right)$ for every choice of $x \in \mathbb{K}$.

It follows that there are two permutations $\alpha$ and $\beta$ of $\mathbb{K}$ such that $(x, y, z)^{\varphi}$ is equal to $\left(x^{\alpha}, y^{\beta}, g_{3}(x, y, z)\right)$. Since $\varphi$ preserves gnarls, it maps the ordinary line $C_{(a, b, c)}$ onto the ordinary line $C_{\left(a^{\alpha}, b^{\beta}, g_{3}(a, b, c)\right)}$. Now notice that the point $(x, y, z)$ can only be contained in the ordinary line $C_{(a, b, c)}$ if $y=b+a^{\theta}(x-a)$. Expressing that the point $(a+x, y, z)$ lies on the circle $C_{(a, b, c)}$ if and only if its image under $\varphi$ lies in $C_{(a, b, c)}^{\varphi}$ shows that, for all $a, b, x \in \mathbb{K}$,

$$
\left(b+a^{\theta} x\right)^{\beta}=b^{\beta}+\left(a^{\alpha}\right)^{\theta}\left((x+a)^{\alpha}-a^{\alpha}\right) .
$$

Putting $b=0$, and noting that $0^{\alpha}=0^{\beta}=0$, we see that $\left(a^{\alpha}\right)^{\theta}\left((x+a)^{\alpha}-\right.$ $\left.a^{\alpha}\right)=\left(a^{\theta} x\right)^{\beta}$, which implies, by substituting this back in Equation (7.1), that $\left(b+a^{\theta} x\right)^{\beta}=b^{\beta}+\left(a^{\theta} x\right)^{\beta}$. So $\beta$ is additive. Put $\ell=1^{\alpha}$. Then we see, by setting $a=1$ and $b=0$ in the Equation (7.1) above, that

$$
x^{\beta}=\ell^{\theta}\left((x+1)^{\alpha}-1^{\alpha}\right),
$$


so $\alpha$ is additive if and only if $(x+1)^{\alpha}=x^{\alpha}+1^{\alpha}$. Plugging in $x=m-1$ in Equation (7.2) we have that $(m-1)^{\beta}=\ell^{\theta}\left(m^{\alpha}-1^{\alpha}\right)$. Because of the additivity of $\beta$ we have on the other hand that $(m-1)^{\beta}=m^{\beta}+(-1)^{\beta}=\ell^{\theta}\left((1+m)^{\alpha}-2 \cdot 1^{\alpha}\right)$. So $\alpha$ is additive as well.

We now have that $x^{\beta}=\ell^{\theta} x^{\alpha}$. We can define the bijection $\sigma: \mathbb{K} \rightarrow \ell^{-1} \mathbb{K}$ : $y \mapsto y^{\sigma}=\ell^{-1} y^{\alpha}$ (note that $1^{\sigma}=1$ ). Plugging in these identities in equation (7.1) yields

$$
\left(b+a^{\theta} x\right)^{\sigma}=b^{\sigma}+\left(a^{\sigma}\right)^{\theta} x^{\sigma},
$$

for all $a, b, x \in \mathbb{K}$. Putting $a=1$, we see that $\sigma$ is additive; putting $b=0$ and $x=1$, we see that $\sigma$ commutes with $\theta$. Putting $b=0$, we see that $(x y)^{\sigma}=x^{\sigma} y^{\sigma}$ for $x \in \mathbb{K}^{\theta}$ and $y \in \mathbb{K}$. If $x, y \in \mathbb{K}$, then

$$
\left((x y)^{\sigma}\right)^{\theta}=\left((x y)^{\theta}\right)^{\sigma}=\left(x^{\theta} y^{\theta}\right)^{\sigma}=\left(x^{\theta}\right)^{\sigma}\left(y^{\theta}\right)^{\sigma}=\left(x^{\sigma}\right)^{\theta}\left(y^{\sigma}\right)^{\theta}=\left(x^{\sigma} y^{\sigma}\right)^{\theta},
$$

and the injectivity of $\theta$ implies that $\sigma$ is an automorphism of $\mathbb{K}$. Now the action of $\varphi$ on a point $(x, y, z)$ is given by $(x, y, z)^{\varphi}=\left(\ell x^{\sigma}, \ell^{1+\theta} y^{\sigma}, g_{3}(x, y, z)\right)$, for all $x, y, z \in \mathbb{K}$.

Let us now investigate what $g_{3}(x, y, z)$ looks like.

The point $p$ with coordinates $\left(a-\frac{a^{\prime}}{a^{\theta}}, 0, a^{\prime \prime}+\left(a^{\prime}-a^{1+\theta}\right)\left(\frac{-a^{\prime}}{a^{\theta}}\right)-\left(\frac{-a^{\prime}}{a^{\theta}}\right)^{2+\theta}\right)$ lies on both $C_{\left(a, a^{\prime}, a^{\prime \prime}\right)}$ and on the ordinary line with gnarl

$$
\left(0,0, a^{\prime \prime}+\frac{\left(a^{1+\theta}-a^{\prime}\right)^{1+\theta}+a^{1+\theta}}{a^{2+\theta}}\right) .
$$

So its image under $\varphi$ lies on the ordinary lines with respective gnarls

$$
\left(\ell a^{\sigma}, \ell^{1+\theta} a^{\prime \sigma}, g_{3}\left(a, a^{\prime}, a^{\prime \prime}\right)\right), \quad\left(0,0, g_{3}\left(0,0, a^{\prime \prime}+\frac{\left(a^{1+\theta}-a^{\prime}\right)^{1+\theta}+a^{1+\theta}}{a^{2+\theta}}\right)\right) .
$$

This leads to

$$
\left\{\begin{aligned}
g_{3}(a & \left.-\frac{a^{\prime}}{a^{\theta}}, 0, a^{\prime \prime}-\frac{\left(a^{\prime}-a^{1+\theta}\right) a^{\prime}}{a^{\theta}}+\left(\frac{a^{\prime}}{a^{\theta}}\right)^{2+\theta}\right) \\
& =g_{3}\left(a, a^{\prime}, a^{\prime \prime}\right)-\ell^{2+\theta}\left(\frac{a^{\prime 2}}{a^{\theta}}-a a^{\prime}-\frac{a^{2+\theta}}{a^{3+2 \theta}}\right)^{\sigma}, \\
g_{3}(a & \left.-\frac{a^{\prime}}{a^{\theta}}, 0, a^{\prime \prime}-\frac{\left(a^{\prime}-a^{1+\theta}\right) a^{\prime}}{a^{\theta}}+\left(\frac{a^{\prime}}{a^{\theta}}\right)^{2+\theta}\right) \\
& =g_{3}\left(0,0, a^{\prime \prime}+\frac{\left(a^{1+\theta}-a^{\prime}\right)^{1+\theta}+a^{1+\theta}}{a^{2+\theta}}\right)-\left(\ell\left(a-\frac{a^{\prime}}{a^{\theta}}\right)^{\sigma}\right)^{2+\theta} .
\end{aligned}\right.
$$

Putting these two equations together we get

$$
\begin{aligned}
g_{3}\left(a, a^{\prime}, a^{\prime \prime}\right)=g_{3}( & \left.0,0, a^{\prime \prime}+\frac{\left(a^{\prime}-a^{1+\theta}\right)^{1+\theta}+a^{\prime+\theta}}{a^{2+\theta}}\right) \\
& -\ell^{2+\theta}\left(\frac{\left(a^{\prime}-a^{1+\theta}\right)^{1+\theta}+a^{\prime+\theta}}{a^{2+\theta}}\right)^{\sigma},
\end{aligned}
$$


for every $a \in \mathbb{K} \backslash\{0\}$ and $a^{\prime}, a^{\prime \prime} \in \mathbb{K}$. We want to extend this equation to one with $a=0$. To this end, we note that the point $\left(0, a^{\prime}, a^{\prime \prime}\right)$ lies on every circle with gnarl $\left(A, a^{\prime}+A^{1+\theta}, a^{\prime \prime}+a^{\prime} A-A^{2+\theta}\right)$, with $A \in \mathbb{K}$. We now only consider $A \neq 0$. Then we take the image under $\varphi$ and obtain that

$$
g_{3}\left(0, a^{\prime}, a^{\prime \prime}\right)=g_{3}\left(A, a^{\prime}+A^{1+\theta}, a^{\prime \prime}+a^{\prime} A-A^{2+\theta}\right)-\ell^{2+\theta}\left(A a^{\prime}-A^{2+\theta}\right)^{\sigma} .
$$

We can now use the above expression for $g_{3}\left(a, a^{\prime}, a^{\prime \prime}\right)$ for $a \neq 0$ to express $g_{3}\left(0, a^{\prime}, a^{\prime \prime}\right)$ in terms of $g_{3}(0,0, z)$, for some $z \in \mathbb{K}$. We carry this out explicitly, and substitute $a^{\prime}=B^{\theta-1}$ and $A=B^{2-\theta}$, and obtain after a tedious calculation

$$
g_{3}\left(0, B^{\theta-1}, a^{\prime \prime}\right)=g_{3}\left(0,0, a^{\prime \prime}-B\right)+\ell^{2+\theta} B^{\sigma},
$$

for all $B \in \mathbb{K} \backslash\{0\}$, and all $a^{\prime \prime} \in \mathbb{K}$. Substituting $-B$ for $B$, we see that $g_{3}\left(0,0, a^{\prime \prime}-B\right)=g_{3}\left(0,0, a^{\prime \prime}+B\right)+\ell^{2+\theta} B^{\sigma}$. We may now put $a^{\prime \prime}=-B$ and finally obtain that $g_{3}(0,0, B)=\ell^{2+\theta} B^{\sigma}$. Plugging this into the formulae above for $g_{3}\left(a, a^{\prime}, a^{\prime \prime}\right), a \neq 0$, and $g_{2}\left(0, a^{\prime}, a^{\prime \prime}\right)$, we see that $g_{3}\left(a, a^{\prime}, a^{\prime \prime}\right)=\ell^{2+\theta} a^{\prime \prime}$, for all $a, a^{\prime}, a^{\prime \prime} \in \mathbb{K}$.

So the action of $\varphi$ on a point $(x, y, z)$ is given by

$$
(x, y, z)^{\varphi}=\left(\ell x^{\sigma}, \ell^{1+\theta} y^{\sigma}, \ell^{2+\theta} z^{\sigma}\right),
$$

with $\sigma$ and $\theta$ commuting automorphisms of $\mathbb{K}$. This action is the restriction to $\Omega$ of the collineation of $\mathrm{H}\left(\mathbb{K}, \mathbb{K}^{\theta}\right)$ defined by the following mapping on the points and lines with five coordinates:

$$
\left\{\begin{array}{l}
\left(a, l, a^{\prime}, l^{\prime}, a^{\prime \prime}\right) \mapsto\left(\ell a^{\sigma}, \ell^{\theta+3} l^{\sigma}, \ell^{\theta+2} a^{\prime \sigma}, \ell^{2 \theta+3} l^{\prime \sigma}, \ell^{\theta+1} a^{\prime \prime \sigma}\right), \\
{\left[k, b, k^{\prime}, b^{\prime}, k^{\prime \prime}\right] \mapsto\left[\ell^{\theta} k^{\sigma}, \ell^{\theta+1} b^{\sigma}, \ell^{2 \theta+3} k^{\prime \sigma}, \ell^{\theta+2} b^{\prime \sigma}, \ell^{\theta+3} k^{\prime \prime \sigma}\right] .}
\end{array}\right.
$$

Now, it is easy to see that $\varphi$ centralizes the polarity $\rho$, and so this completes the proof of our Main Result 1.

\section{Automorphism group of the truncated Ree geometry restricted to points and circles}

General idea. Let $\mathscr{C}_{\mathcal{C}}=(\mathcal{P}, \mathcal{C})$ be the truncated Ree geometry, with $\mathscr{C}$ the set of circles. We first prove that the gnarls of circles have to be mapped onto gnarls of circles. Then we use the result from the previous section to prove that the automorphism group of $\mathscr{E} \mathcal{E}$ is equal to the automorphism group of the Ree geometry $\mathscr{E}$. 
We denote by $\mathscr{E}^{\prime}$ the derived geometry in $(\infty)$ (so the point set is $\mathcal{P} \backslash\{(\infty)\}$ and the blocks are the vertical and ordinary lines, as defined in Section 6.1).

Lemma 8.1. The full group $G$ of automorphisms of $\mathscr{E}^{\prime}$ has two orbits on the lines, which are the vertical and the ordinary lines.

Proof. It is clear that $G$ acts transitively on both the set of vertical lines and the set of ordinary lines (as $G$ contains the corresponding Ree group), so we only have to exclude the possibility of one orbit. We suppose this is the case and derive a contradiction.

Consider, as previously, the point set $\mathcal{P} \backslash\{(\infty)\}$ as a 3-dimensional affine space with point set $\left\{\left(a, a^{\prime}, a^{\prime \prime}\right) \mid a, a^{\prime}, a^{\prime \prime} \in \mathbb{K}\right\}$. We project it on the 2-dimensional space $\left\{\left(a, a^{\prime}, 0\right) \mid a, a^{\prime} \in \mathbb{K}\right\}$ by the standard projection map $\left(a, a^{\prime}, a^{\prime \prime}\right) \mapsto$ $\left(a, a^{\prime}, 0\right)$. The projection of a vertical line $L_{a, a^{\prime}}$ is the point $\left(a, a^{\prime}, 0\right)$, and the projection of an ordinary line $C_{\left(a, a^{\prime}, a^{\prime \prime}\right)}$ is the affine line $Y=a^{\theta} X+\left(a^{\prime}-a^{1+\theta}\right)$. The images of a parallel class of ordinary lines will form a parallel class of affine lines in the 2-dimensional space. This implies that all these affine lines form the line set of a net $\mathcal{N}$, and a parallel class of ordinary lines is projected to a parallel class in this net. (A net is a point-line geometry where for each point $p$ not on a line $L$, there exist an unique line incident with $p$, parallel with $L$.)

Let $L$ be a vertical line and $M$ a vertical or ordinary line disjoint from $L$. If $M$ is a vertical line then the projection of $L$ and $M$ are two points. If there exists one ordinary line such that the projection contains both points, then translating this back to the lines means through each point of $L$ there is an (ordinary) line intersecting $M$ (by varying the third coordinate $a^{\prime \prime}$ ). If, on the other hand, there is no projection of an ordinary line containing both points, then there is no (ordinary) line intersecting both $L$ and $M$.

If $M$ is an ordinary line, then the projection of $M$ is a certain affine line with equation $Y=a^{\theta} X+\left(a^{\prime}-a^{1+\theta}\right)$. As no projection of an ordinary line is of the form $X=c$ with $c \in \mathbb{K}$ a constant, there are points of $M$ through which no (ordinary) line passes that also intersects $L$ (because we would have projections of the form $X=c$ ). Also, there obviously are ordinary lines whose projection contains the projection of $L$ and intersect the projection of $M$. The set of ordinary lines projected to this projection forms a subset of a parallel class exactly one member of which intersects both $L$ and $M$. We conclude that there exist lines intersecting both $L$ and $M$, but not through each point of $M$.

In the above two paragraphs we proved that we can tell a vertical line from an ordinary line if one vertical line is given. Using the hypothesis that there is only one orbit on the lines, this implies that there is an equivalence relation on the lines which is preserved by $G$. One of the equivalence classes is obviously the set of 
vertical lines. By transitivity it follows that through each point of $\mathscr{E}^{\prime}$ there is exactly one line of a given equivalence class. We now claim that the other classes are the parallel classes of ordinary lines. Indeed, if an ordinary line $C_{\left(a, a^{\prime}, a^{\prime \prime}\right)}$ lies in a certain equivalence class, then all lines $C_{\left(a, a^{\prime}, k\right)}$ with $k \in \mathbb{K}$ lie in this class, because there is a vertical line through each point of $C_{\left(a, a^{\prime}, a^{\prime \prime}\right)}$ intersecting $C_{\left(a, a^{\prime}, k\right)}$. The subsets of the equivalence classes thus obtained are exactly the set of lines projected to a common affine line. As two intersecting affine lines can be viewed as the projection of two intersecting ordinary lines, two of these subsets are parallel if and only if the corresponding affine lines are parallel. This implies that the equivalence classes are subpartitions of the parallel classes. But since through each point there has to be a line of each equivalence class, the latter must coincide with a parallel class.

Now consider the ordinary line $C_{(0,0,0)}$ and its parallel class $\pi$. We can conjugate the center of $U_{(\infty)}$ to obtain an automorphism $\phi \in G$ that fixes the ordinary lines in $\pi$, acts freely on the points of such a line, fixes the equivalence classes, and maps $(0,0,0)$ to $(1,0,-1)$.

Let $\left(x, x^{\prime}, x^{\prime \prime}\right)$ be an arbitrary point of $\mathscr{e}^{\prime}$. This point lies on the ordinary line $C_{\left(0, x^{\prime}, b\right)}=\left\{\left(t, x^{\prime}, b+x^{\prime} t-t^{2+\theta}\right) \mid t \in \mathbb{K}\right\}$ for $t=x$ with $b:=x^{\prime \prime}-x^{\prime} x+x^{2+\theta}$. As this ordinary line is an element of $\pi$, the point $\left(x, x^{\prime}, x^{\prime \prime}\right)^{\phi}$ also lies on this line. Hence there exists an $f_{x^{\prime}, b}(x) \in \mathbb{K}$ such that $\left(x, x^{\prime}, x^{\prime \prime}\right)^{\phi}=\left(f_{x^{\prime}, b}(x), x^{\prime}\right.$, $\left.b+x^{\prime} f_{x^{\prime}, b}(x)-f_{x^{\prime}, b}(x)^{2+\theta}\right)$. Notice that the middle coordinate is always fixed.

The vertical line $L_{x, x^{\prime}}=\left\{\left(x, x^{\prime}, t\right) \mid t \in \mathbb{K}\right\}$ must be mapped to another vertical line $L_{f_{x^{\prime}, b}(x), x^{\prime}}=\left\{\left(f_{x^{\prime}, b}(x), x^{\prime}, t\right) \mid t \in \mathbb{K}\right\}$. From this it follows that the function $f$ is independent from the the last coordinate. As both the first and second coordinate are independent from the last, it follows that $\phi$ induces an automorphism $\phi^{\prime}$ on the net $\mathcal{N}$, mapping $\left(x, x^{\prime}, 0\right)$ to $\left(f_{x^{\prime}, b}(x), x^{\prime}, 0\right)$. Now $\phi^{\prime}$ also fixes every parallel class of $\mathcal{N}$ (the parallel class coming from $\pi$ is even fixed linewise) and maps $(0,0,0)$ to $(1,0,0)$ (because $\left.(0,0,0)^{\phi}=(1,0,-1)\right)$. It is now easy to see that this implies $f_{x^{\prime}, b}(x)=x+1$. This gives us the following explicit formula for $\phi$ :

$$
\begin{aligned}
\phi:\left(x, x^{\prime}, x^{\prime \prime}\right) & \mapsto\left(x+1, x^{\prime}, x^{\prime \prime}-x^{\prime} x+x^{2+\theta}+x^{\prime}(x+1)-(x+1)^{2+\theta}\right) \\
& \mapsto\left(x+1, x^{\prime}, x^{\prime \prime}+x^{\prime}+x^{2+\theta}-(x+1)^{2+\theta}\right) .
\end{aligned}
$$

The image of the ordinary line $C_{(1,1,0)}=\left\{\left(1+t, 1+t,-t^{2+\theta}\right) \mid t \in \mathbb{K}\right\}$, using the formula for $\phi$, is

$$
C_{(1,1,0)}^{\phi}=\left\{\left(t-1, t+1,-t^{2+\theta}-t^{2}+t^{1+\theta}+t\right) \mid t \in \mathbb{K}\right\} .
$$

This has to coincide with a certain ordinary line $C_{\left(1, a^{\prime}, a^{\prime \prime}\right)}=\left\{\left(1+s, a^{\prime}+s\right.\right.$, $\left.\left.a^{\prime \prime}+\left(a^{\prime}-1\right) s-s^{2+\theta}\right) \mid s \in \mathbb{K}\right\}$ (because the parallel class is preserved) with 
$a^{\prime}, a^{\prime \prime} \in \mathbb{K}$. This yields the following system of equalities:

$$
\left\{\begin{array}{l}
t-1=1+s, \\
t+1=a^{\prime}+s, \\
-t^{2+\theta}-t^{2}+t^{1+\theta}+t=a^{\prime \prime}+\left(a^{\prime}-1\right) s-s^{2+\theta},
\end{array}\right.
$$

which simplifies to

$$
\left\{\begin{array}{l}
s=t+1, \\
a^{\prime}=0, \\
t=a^{\prime \prime}+1-t^{\theta} .
\end{array}\right.
$$

If $t=0$ the last equation gives us $a^{\prime \prime}=-1$, but if we use $t=1$, we obtain $a^{\prime \prime}=1$, which is a contradiction as $a^{\prime \prime}$ is a constant. It follows that the hypothesis of one orbit is false.

The following corollary follows directly:

Corollary 8.2. The gnarls of circles are mapped onto gnarls of circles.

Using the above and Lemma 6.1, one can reconstruct the spheres, giving the following result (which is part of Main Result 2):

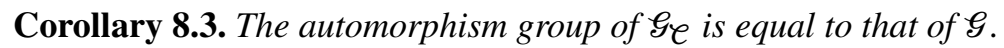

\section{Absolute points and lines of polarities in the Ree hexagon}

We now show our Main Corollary in the formulation below. We note that our proof will not use the full strength of our results proved so far. Indeed, we will only use Corollary 8.2. The last few lines of the proof of the next corollary can be deleted if we use Main Result 1.

Corollary 9.1. If a collineation $\sigma$ of a Moufang hexagon stabilizes the set of all absolute points of some polarity, then it stabilizes the set of all absolute lines as well.

Proof. By Theorem 7.3.4 and Theorem 7.7.2 of [14], any polarity $\rho$ of a Moufang hexagon is associated to a Ree group, so it is a polarity of the associated Ree hexagon.

As mentioned before, a circle $C$ of the Ree geometry is the set of absolute points at distance 3 from a line $M$, not going through an absolute point. The collineation $\sigma$ maps this set to the set of absolute points at distance 3 from $M^{\sigma}$, which is again a circle since $M^{\sigma}$ clearly is not incident with any absolute point (as $\sigma$ stabilizes the set of absolute points). It follows that $\sigma$ induces an automorphism of $\mathscr{E e}_{\text {e }}$. 
The gnarl of $C$ is the absolute point $x$ such that the corresponding absolute line $x^{\rho}$ intersects $M$. Corollary 8.2 now implies that the absolute line $\left(x^{\sigma}\right)^{\rho}$ intersects $M^{\sigma}$. As $\left(x^{\rho}\right)^{\sigma}$ also contains $x^{\sigma}$ and intersects $M^{\sigma}$, it follows that $\left(x^{\sigma}\right)^{\rho}=\left(x^{\rho}\right)^{\sigma}$. This means that the absolute line $x^{\rho}$ is mapped to another absolute line. Varying $C$ we now see that the set of all absolute lines is stabilized by $\sigma$.

\section{Automorphism group of the truncated Ree geometry restricted to points and spheres}

General idea. Let $\mathscr{E}_{\delta}=(\mathcal{P}, \delta)$ be the truncated Ree geometry with $\delta$ the set of spheres. We again prove that the gnarls of spheres have to be mapped onto gnarls of spheres. As a consequence one can recognize certain automorphisms of the Ree geometry generating the Ree group, from which the circles can be reconstructed giving us the full Ree geometry $\mathcal{E}$ and its automorphism group.

We denote by $\mathscr{E}_{\mathcal{S}^{\prime}}$ the derived geometry in $(\infty)$ (so the point set is $\mathcal{P} \backslash\{(\infty)\}$ and the blocks are the vertical and ordinary planes, as defined in Section 6.1).

We start with some small observations:

Lemma 10.1. A vertical plane and an ordinary plane always intersect.

Proof. By transitivity we can suppose that the vertical plane is given by

$$
P_{a}=\left\{\left(a, t^{\prime}, t^{\prime \prime}\right) \mid t^{\prime}, t^{\prime \prime} \in \mathbb{K}\right\}, \text { with } a \in \mathbb{K}
$$

and the ordinary plane by $S_{(0,0,0)}$, which is the set

$$
\begin{aligned}
\left\{\left(\frac{x^{\prime \prime \theta}-x^{\prime} x^{\prime \prime}}{x^{\prime \prime 2}+x^{\prime 1+\theta}}, \frac{-x^{\prime \theta}}{x^{\prime \prime 2}+x^{\prime 1+\theta}}, \frac{-x^{\prime \prime}}{x^{\prime \prime 2}+x^{\prime 1+\theta}}\right) \mid \mathbb{K} \times \mathbb{K} \ni\left(x^{\prime}, x^{\prime \prime}\right) \neq(0,0)\right\} \\
\cup\{(0,0,0)\} .
\end{aligned}
$$

If $a=0$, then $(0,0,0) \in P_{a} \cap S_{(0,0,0)}$. If $a \neq 0$, then, putting $x^{\prime}=0$ and $x^{\prime \prime}=a^{-2-\theta}$ in the formula of $S_{(0,0,0)}$ gives the point $\left(a, 0,-a^{2+\theta}\right)$, which is also a point of $P_{a}$.

Lemma 10.2. The intersection of $P_{0}$ and $S_{(0,0,0)}$ is given by the set $\{(0, t, 0) \mid t \in$ $K\} \cup\left\{\left(0, t^{\theta-1}, t\right) \mid t \in \mathbb{K} \backslash\{0\}\right\}$.

Proof. Using the representations of $P_{0}=\left\{\left(0, t, t^{\prime}\right) \mid t, t^{\prime} \in \mathbb{K}\right\}$ and

$$
\begin{aligned}
S_{(0,0,0)}=\left\{\left(\frac{x^{\prime \prime \theta}-x^{\prime} x^{\prime \prime}}{x^{\prime \prime 2}+x^{\prime 1+\theta}}, \frac{-x^{\prime \theta}}{x^{\prime \prime 2}+x^{\prime 1+\theta}}, \frac{-x^{\prime \prime}}{x^{\prime \prime 2}+x^{\prime 1+\theta}}\right) \mid\right. \\
\left.\mathbb{K} \times \mathbb{K} \ni\left(x^{\prime}, x^{\prime \prime}\right) \neq(0,0)\right\} \cup\{(0,0,0)\},
\end{aligned}
$$


we see that the points of the intersection are determined by the equation $x^{\prime \prime \theta}-$ $x^{\prime} x^{\prime \prime}=0$. The solutions of this equation are given by $x^{\prime \prime}=0$ or $x^{\prime}=x^{\prime \prime \theta-1}$. The first set of solutions gives us $\{(0, t, 0) \mid t \in K\}$, the second $\left\{\left(0, t^{\theta-1}, t\right) \mid t \in\right.$ $\mathbb{K} \backslash\{0\}\}$.

Note that $P_{0}$ is the disjoint union of affine unital blocks. Indeed, the affine blocks $\{(0, t, b) \mid t \in \mathbb{K}\}$, with $b \in \mathbb{K}$, partition $P_{0}$. It is now clear that the intersection of $S_{(0,0,0)}$ and $P_{0}$ contains exactly one affine unital block, and all other affine unital blocks in $P_{0}$ share exactly one point with that intersection.

Lemma 10.3. The ordinary planes $S_{(0,0,0)}$ and $S_{\left(0, a^{\prime}, a^{\prime \prime}\right)}$ with $a^{\prime}, a^{\prime \prime} \in \mathbb{K}$ intersect.

Proof. Since $\left(0, a^{\prime}, a^{\prime \prime}\right) \in U_{\infty}$ maps $P_{0}$ to itself and $S_{(0,0,0)}$ to $S_{\left(0, a^{\prime}, a^{\prime \prime}\right)}$, it follows from the paragraph preceding this lemma that $P_{0} \cap S_{\left(0, a^{\prime}, a^{\prime \prime}\right)}$ contains an affine unital block $B$. But from that same paragraph also follows that $B$ shares a point with $P_{0} \cap S_{(0,0,0)}$. That point is hence contained in $S_{(0,0,0)} \cap S_{\left(0, a^{\prime}, a^{\prime \prime}\right)}$.

The above lemmas now allow us to prove the following analogue to Lemma 8.1.

Lemma 10.4. The full group $G$ of automorphisms of $\mathscr{E}_{8}^{\prime}$ has two orbits on the planes, which are the vertical and the ordinary planes.

Proof. As with the case of points and circles, it suffices to prove that the planes cannot be all in one orbit. So suppose this is the case.

We call two vertical or ordinary planes parallel if they are disjoint or equal. By the transitivity assumption on the planes and Lemma 10.1, for each point $p$ (different from $(\infty)$ ) and plane $P$, there is exactly one plane $Q$ parallel to $P$ and containing $p$. Let $\varpi$ be the parallel class where $S_{(0,0,0)}$ belongs to. Because $U_{\infty}$ preserves parallelism and acts regularly on the ordinary planes, the stabilizer $V$ of $\varpi$ in $U_{\infty}$ acts regularly on the planes in $\varpi$ and $S_{\left(a, a^{\prime}, a^{\prime \prime}\right)} \in \varpi$ if and only if $\left(a, a^{\prime}, a^{\prime \prime}\right) \in V$.

Let $g=\left(a, a^{\prime}, a^{\prime \prime}\right) \in U_{\infty}$ be a non-trivial element of $V$. Then, in view of Lemma $10.3, a$ has to be different from 0 . But as $V$ is a group, $g^{3}=\left(0,0,-a^{2+\theta}\right)$ is also a non-trivial element of $V$, which does have as first coordinate 0 , so the hypothesis is false.

Lemma 10.5. In $\mathcal{E}_{s}^{\prime}$ the affine unital blocks are (geometric) invariants.

Proof. We will denote the intersection of a vertical plane through the point $p$ with the ordinary plane with gnarl $p$ by $W_{p}$. The sets $W_{p}$ are invariants of the geometry 
by virtue of Lemma 10.4. Lemma 10.2 implies that the affine unital block through $p$ is contained in $W_{p}$.

By transitivity, it suffices to construct the affine unital block $B$ through $(0,0,0)$. Let $p \in W_{(0,0,0)}$ be a point different from $(0,0,0)$. If $p$ lies on $B$, then $W_{(0,0,0)} \cap$ $W_{p}$ contains $B$ itself and thus at least 4 points (as $|\mathbb{K}|>3$ ). Now suppose $p \notin B$, so $p=\left(0, k^{\theta-1}, k\right)$ for a certain $k \in \mathbb{K}$ different from 0 . Using $\left(0, k^{\theta-1}, k\right)$ as an element of $U_{\infty}$ and Lemma 10.2 we calculate that $W_{p}=\left\{\left(0, t+k^{\theta-1}, k\right) \mid\right.$ $t \in \mathbb{K}\} \cup\left\{\left(0, t^{\theta-1}+k^{\theta-1}, t+k\right) \mid t \in \mathbb{K} \backslash\{0\}\right\}$. The intersection $W_{(0,0,0)} \cap W_{p}$ contains two obvious intersection points on the affine unital blocks contained in either $W_{(0,0,0)}$ and $W_{p}$. To look for more intersection points we need to investigate whether or not it is possible to have $\left(0, t^{\theta-1}+k^{\theta-1}, t+k\right)=\left(0, s^{\theta-1}, s\right)$ for certain $s, t \in \mathbb{K} \backslash\{0\}$. Equality on the third coordinate gives us $t+k=s$, the second gives us

$$
\begin{aligned}
s^{\theta-1}=t^{\theta-1}+k^{\theta-1} & \Leftrightarrow(t+k)^{\theta-1}=t^{\theta-1}+k^{\theta-1} \\
& \Leftrightarrow t^{2-\theta}=-k^{2-\theta} .
\end{aligned}
$$

If we raise both hand sides of the last equation to the power $2+\theta$, then we obtain $t=-k$, implying $s=0$, a contradiction.

Thus in this case we have that $\left|W_{(0,0,0)} \cap W_{p}\right|=2$. This allows us to recognize the points of the affine unital block through $(0,0,0)$ as those for which $\mid W_{(0,0,0)} \cap$ $W_{p} \mid>2$.

Lemma 10.6. In $\mathscr{E}_{\boldsymbol{g}}$, the circles of $\mathcal{E}$ are invariants.

Proof. Let $p$ and $q$ be two different points of $\mathscr{E}_{8}$ and put $G$ equal to the full automorphism group of $\mathscr{G}_{\S}$. Then we first want to determine the elements of $G$ which fix $p$ and all the blocks of the unital through $p$ within the sphere with gnarl $p$ through $q$. We will denote this group by $G_{[p, q[}$.

By 2-transitivity we can suppose that $p=(\infty)$ and $q=(0,0,0)$. The aim is to prove that $G_{[(\infty),(0,0,0)[}=\{(0, t, 0) \mid t \in \mathbb{K}\}=: H$. It is easy to see that these automorphisms satisfy the needed properties and act transitively (even regularly) on the points of the affine unital block $B$ through $(0,0,0)$. Suppose there is another automorphism $g$ which satisfies the properties. Then, possibly by composing with a suitable element of $H$, we may assume that $g$ fixes $(0,0,0)$. This implies that the sphere with gnarl $(0,0,0)$ through $(\infty)$ is also fixed. By Lemma 10.2 the points $\left(0, k^{\theta-1}, k\right)$ with $k \in \mathbb{K} \backslash\{0\}$ are also fixed, thus also the blocks through $(0,0,0)$ in the sphere with gnarl $(0,0,0)$ through $(\infty)$, which makes the situation symmetric in both points. We can also let the fixed points of the form $\left(0, k^{\theta-1}, k\right)$ play the role of $(0,0,0)$, which yields the fixed points $\left(0, k_{1}^{\theta-1}+k_{2}^{\theta-1}+\cdots+k_{n}^{\theta-1}, k_{1}+k_{2}+\cdots+k_{n}\right)$ with $k_{i} \in \mathbb{K} \backslash\{0\}$ by repeating 
the argument. Choosing $n=3$ and $k_{1}=-k_{2}=k_{3}=k$ with $k \in \mathbb{K} \backslash\{0\}$ gives us the fixed points $(0,0, k)$ for all $k \in \mathbb{K}$.

Interchanging the roles of the points $(\infty)$ and $(0,0,0)$, we obtain the fixed points $\left(k, 0,-k^{2+\theta}\right)$ (to calculate these observe that $(0,0, k)$ are the points different from $(\infty)$ on the circle with gnarl $(\infty)$ through $(0,0,0)$, interchanging gives us the points different from $(0,0,0)$ on the circle with gnarl $(0,0,0)$ through $(\infty))$. If we let a fixed point $(0,0, l)$ with $l \in \mathbb{K}$ play the role of $(0,0,0)$, we obtain that all the points of the form $(k, 0, l)$ with $k, l \in K$ are fixed points. On each affine unital block lies a point of this form, so all affine unital blocks are fixed, and by symmetry also the blocks of the Ree unital through $(0,0,0)$. It follows that all points are fixed points, and that $g$ is the identity.

The above proves that $G_{[p, q[}$ is a subgroup of the root group $U_{p}$ and hence, if $|\mathbb{K}|>3$, also a subgroup of the simple Ree group $\mathrm{R}^{\prime}(\mathbb{K}, \theta)$. The group $K$ generated by all groups of the form $G_{[p, q[}$ is a normal subgroup of this Ree group (indeed, if $g$ is a automorphism of $\mathscr{G}_{8}$, then $G_{[p, q[}^{g}=G_{\left[p^{g}, q^{g}[\right.}$ ). By simplicity, $K$ coincides with $\mathrm{R}^{\prime}(\mathbb{K}, \theta)$. Now, by [1], the root groups of $K$ are the unique unipotent subgroups of $K$. Hence we can recover these root groups and consequently also the circles constructed from these root groups.

If $|\mathbb{K}|=3$, then $K$ is a normal subgroup of the Ree group R(3) over the field with 3 elements. But the groups $G_{[p, q \text { [ }}$ do not belong to the simple Ree group. Hence, it is easy to see that $K$ coincides with the Ree group R(3) and, as above, we can again recover the circles.

We have proved:

Corollary 10.7. The automorphism group of $\mathscr{E}_{8}$ coincides with that of $\mathscr{E}$.

This completes the proof of Main Result 2.

\section{Bibliography}

[1] T. De Medts, F. Haot, R. Knop and H. Van Maldeghem, On the uniqueness of the unipotent subgroups of some Moufang sets, in: Finite Geometries, Groups, and Computation, pp. 43-66, Walter de Gruyter, Berlin, 2006.

[2] T. De Medts and H. Van Maldeghem, Moufang sets of type $\mathrm{F}_{4}$, submitted to Mathematische Zeitschrift.

[3] V. De Smet and H. Van Maldeghem, The finite Moufang hexagons coordinatized, Beiträge Algebra Geom. 34 (1993), 217-232.

[4] H. Lüneburg, Some remarks concerning the Ree groups of type $\left(G_{2}\right)$, J. Algebra 3 (1966), 256-259. 
[5] B. Mühlherr and H. Van Maldeghem, Moufang sets from groups of mixed type, J. Algebra 300 (2006), 820-833.

[6] R. Ree, A family of simple groups associated with the Lie algebra of type $\left(G_{2}\right)$, Amer. J. Math. 83 (1961), 432-462.

[7] M. Stroppel and H. Van Maldeghem, Automorphisms of unitals, Bull. Belg. Math. Soc. Simon Stevin 12 (2005), 895-908.

[8] K. Struyve, Moufang sets related to polarities in exceptional Moufang quadrangles of type $\mathrm{F}_{4}$, submitted to Innov. Incid. Geom.

[9] J. A. Thas, K. Thas and H. Van Maldeghem, Translation Generalized Quadrangles, World Scientific, 2006.

[10] J. Tits, Sur la trialité et certains groupes qui s'en déduisent, Inst. Hautes Études Sci. Publ. Math. 2 (1959), 13-60.

[11] J. Tits, Les groupes simples de Suzuki et de Ree, Séminaire Bourbaki 13 (1960/61), no. $210,1-18$.

[12] J. Tits, Buildings of Spherical Type and Finite BN-Pairs, Lecture Notes in Mathematics 386, Springer-Verlag, Berlin, Heidelberg, New York, 1974.

[13] J. Tits, Résumé de cours (Annuaire du Collège de France), 97e année, 1996-1997, pp. 89-102.

[14] H. Van Maldeghem, Generalized Polygons, Monographs in Mathematics 93, Birkhäuser-Verlag, Basel, Boston, Berlin, 1998.

[15] H. Van Maldeghem, Moufang lines defined by (generalized) Suzuki groups, European J. Combin. 28 (2007), 1878-1889.

Received January 4, 2008.

\section{Author information}

Fabienne Haot, Department of Pure Mathematics and Computer Algebra, Ghent University, Krijgslaan 281, S22, 9000 Gent, Belgium.

E-mail: fabiennehaot@gmail.com

Koen Struyve, Department of Pure Mathematics and Computer Algebra, Ghent

University, Krijgslaan 281, S22, 9000 Gent, Belgium.

E-mail: kstruyve@cage.UGent. be

Hendrik Van Maldeghem, Department of Pure Mathematics and Computer Algebra,

Ghent University, Krijgslaan 281, S22, 9000 Gent, Belgium.

E-mail: hvm@cage.UGent. be 